\title{
Implementing Cardiac Testing to Mitigate the Risk of Sudden Cardiac Death: An Evidence-to-Practice Review
}

Victoria J. Simpson, MS, ATC*; Zachary K. Winkelmann, PhD, SCAT, ATC*

*University of South Carolina, Columbia, SC

\begin{abstract}
In the United States, sudden cardiac death (SCD) is the leading cause of fatality in young athletes during exercise. Allied healthcare professionals have used screening tools such as the 12-Element American Heart Association recommendations, the European Society of Cardiology (ESC) recommendations, and the International Olympic Committee guidelines to screen for cardiovascular abnormalities in athletes. Although each of these protocols consist of personal and family history questions complemented with a physical examination, a uniform screening strategy to identify athletes at risk of SCD does not exist. The purpose of this evidence to practice review is to summarize a systematic review regarding SCD-related abnormalities in young Middle Eastern and African competitive athletes and to assess available cardiac preparticipation physical examination (PPE) screening tools. The authors examined literature that reported the prevalence of positive cardiac abnormalities detected with history and physical examination tools compared to noninvasive cardiac testing, such as electrocardiography. The review also highlights the significant number of false positives achieved through history and physical examination tools only, whereas electrocardiography interpretations indicated by the 2014 Refined Criteria are superior to the 2013 Seattle Criteria and the 2010 ESC Recommendations. The guiding systematic review indicates that electrocardiography is sensitive and specific to predicting SCD-related abnormalities and should be a tool implemented in the PPE.
\end{abstract}

\section{Key Phrases}

Public health, clinician-rated outcomes, patientrated outcomes, professional standards

\section{Correspondence}

Dr. Zachary Winkelmann, 1300 Wheat Street, Columbia, SC 29208.

E-mail:winkelz@mailbox.sc.edu

Twitter: @zachwinkelmann

\section{Full Citation}

Simpson VJ, Winkelmann ZK. Implementing cardiac testing to mitigate the risk of sudden cardiac death: An evidence-to-practice review. Clin Pract Athl Train. $2021 ; 4(1)$ : 36-42.

https://doi.org/10.31622/2021/0004.1.5.

Submitted: December 15, 2020 Accepted: April 4, 2021.

Copyright (C) by Indiana State University All rights reserved. ISSN Online 2577-8188

\section{ORIGINAL REFERENCE}

Hallak YO, Battistin U, Al-Masaeed AM. Cardiac screening to mitigate the risk of sudden cardiac death in Middle Eastern and African competitive athletes. A systematic review. J Saudi Heart Assoc. 2020; 32(2): 174- 185.

\section{SUMMARY}

\section{CLINICAL PROBLEM AND QUESTION}

P rior to a competitive season, athletes undergo a preparticipation physical examination (PPE). The PPE includes vital screening tools utilized by allied healthcare professionals to determine readiness for a specific sport and identify any potential or correctable conditions that may impair athletic performance. ${ }^{1}$ The National Athletic Trainers' Association (NATA) has compiled recommendations for items included in a PPE which includes personal and family medical history, current medical conditions, signs and symptoms during exercise, and system-based physical examinations (e.g., cardiovascular, central nervous system, pulmonary). ${ }^{2}$ Currently, the NATA position statement recommends "noninvasive cardiac testing (e.g., echocardiography, ECG, exercise stress testing) not be a routine aspect of PPE screening unless warranted by findings from personal and family history". 2 This position statement also indicated the need for continued research on cardiac screening methods to "improve the ability to identify at-risk individuals in a cost-effective manner" with an acceptable false-positive rate to be utilized as a screening method to reduce the rates of sudden cardiac death $(\mathrm{SCD}){ }^{3}$ 
In the United States between 2014-2018,50\% of sudden cardiac arrest cases occurred in Caucasian competitive athletes with $55 \%(n=88 / 164)$ of those cases resulting in survival.4 However, the survival rates for African American athletes was only $25 \%(n=39 / 109)$ with an accompanied incidence rate for a male, African American, National Collegiate Athletic Association (NCAA) Division 1 basketball player at 1:2087.4 The rates in male, African American athletes are 2.4 times higher when compared to their Caucasian counterparts. 1 The NCAA also reports the incidence of $S C D$ as higher in Black athletes $(1: 1700)$ and male basketball players $(1: 7000) .{ }^{5}$ Reports in the United States have estimated an incidence of 110 SCD cases each year in young athletes, which equates to 1 death every 3 days. ${ }^{5}$ Additionally, the literature supports that African American/Black athletes are 5 times more likely to develop hypertrophic cardiomyopathy (HCM), which is the leading cause of SCD in young competitive athletes when compared to their Arab counterparts. ${ }^{6}$

The scarcity of literature reporting the prevalence rates of SCD in the Middle East and Africa highlight the lack of ethnic-specific cardiac PPE screenings in these regions. Therefore, there is a need to exploring equitable screening and testing practices dependent upon race/ethnicity. The purpose of this evidence-to-practice review was to assess cardiac PPE screening methods and their ability to detect predisposing abnormalities correlated with SCD in young Middle Eastern and African competitive athletes.

\section{SUMMARY OF LITERATURE}

The guiding systematic review's authors conducted a systematic search with PubMed (MEDLINE) and Google Scholar that examined the findings of Middle Eastern and African competitive athletes using any cardiac PPE screening method. The authors applied the Preferred Reporting Items for Systematic Reviews and Meta-Analyses (PRISMA)

Copyright $(\subseteq$ by Indiana State University All rights reserved. ISSN Online 2577-8188 guidelines to conduct their search. The studies included in the systematic review had to meet the following inclusion criteria: (1) original published studies in peer-reviewed journals, in English or Arabic, involving PPE cardiac screening in Arab or African competitive athletes, (2) study population not restricted to gender, or athletic level, but age restricted to less than 36 years of age, (3) involved no less than 100 participants as sample size, (4) included cardiac screening programs and African or Middle Eastern Athletes, and (5) articles published between January 2009 and March 2019.

The PubMed (MEDLINE) and Google Scholar database searches identified 17 articles that met the initial inclusion criteria. Studies were eventually excluded due to their title, abstract screening, non-athletic populations, studies conducted outside of Arab or African competitive athletes, or if they were a book, editorial, case report, or review. The number of papers were reduced to 6 prior to the authors conducting a full text screening for eligibility. Overall, 4 studies were included in the qualitative synthesis of this systematic review. The inclusion of only 4 studies in this systematic review diminished the authors potential to generalize this data, yet this discrepancy depicts the gap in literature focused on PPE screening in athletes in this region of the world. The regions where these articles were conducted include 2 studies in Qatar, 1 study in the United Arab Emirates, and 1 study in Gabon. The authors noted that the main limitation of this study was the restricted access to only 2 databases, which may have decreased their yielded results.

\section{SUMMARY OF OUTCOMES}

The authors of the guiding systematic review gathered the sample characteristics, sample size and population, study setting and focus, screening tools, prevalence of cardiovascular abnormalities, and personal and family history from the 
participants in the 4 studies. The authors designated the 2010 European Society of Cardiology (ESC) as the guideline used to interpret ECG findings amongst the studies. The study conducted by Alattar et al.7 (listed as Maffulli $\mathrm{N}$ et al. in the guiding systematic review) incorporated the Lausanne recommendations along with the ESC for interpretation of United Arab Emirates National athletes. This method includes collection of personal and family history, a physical examination, and a 12-lead ECG.7 The abnormalities associated with SCD that were examined in these studies included WolffParkinson-White syndrome (WPW), arrhythmogenic right ventricular cardiomyopathy (ARVC), HCM, dilated cardiomyopathy, and long QT syndrome.

\section{FINDINGS AND CLINICAL IMPLICATIONS}

The guiding systematic review assessed the reported prevalence of cardiac abnormalities with the efficacy of various cardiac screening methods for Middle Eastern and African athletes. The information from the 4 studies compiled to a total of 3,655 men, with ages ranging from 12 to 35 years, who participated in a variety of sports including: football, basketball, tennis, volleyball, and handball. All studies incorporated the ESC recommendations to assess the results of cardiovascular screenings of participants.

There were variations in results pertaining to the prevalence of these SCD-related abnormalities, which ranged from $0.47 \%-4.29 \%$ across all studies. Specifically, Alattar et al.7 reported 5 Arab athletes $(2.17 \%)$ with SCD-related abnormalities including 2 with WPW, 1 with atrial fibrillation, 1 with Long QT syndrome, and 1 with ARVC. Riding et al. 6 documented SCD-related abnormalities in $10(0.47 \%)$ athletes: 5 Black and 2 Arab athletes with $\mathrm{HCM}$, and 3 Arab athletes with WPW. Wilson et al. 8 recorded $7(0.63 \%)$ athletes with an SCD-associated abnormality: 1 Arab and 1 African athlete had WPW, 2 Arab

Copyright $\left({ }^{\circ}\right.$ by Indiana State University All rights reserved. ISSN Online 2577-8188 and 1 African athlete had HCM, 1 African athlete had ARVC, and 1 Arab athlete had Long QT syndrome. The final study by Schmied et al. 9 detailed those 9 (4.29\%) African athletes had an SCD-related abnormality: 5 with Long QT syndrome, 3 with WPW, and 1 with HCM upon echocardiography.

The prevalence of SCD-related abnormalities in these Arab athletes were $2.17 \%$ and produced false-positive rates at $20.4 \% .7$ The Riding et al. 6 study examined false-positive rates when using the ESC, Seattle Criteria, and the 2014 Refined Criteria in Arab and Black athletes. The ECG interpretations by the 2014 Refined Criteria decreased false-positives to $3.6 \%$ in Arab athletes (vs 19.1\% using ESC guidelines and 9.7 $\%$ using Seattle criteria) and $10 \%$ in Black athletes (vs 29.9\% using ESC guidelines and $16.6 \%$ using Seattle criteria). 6 The 2014 Refined Criteria was $100 \%$ sensitive in discovering SCDrelated cardiac pathologies, while reducing falsepositives. 6

Alattar et al. ${ }^{7}$ indicated 2 Arab athletes had a positive family history of SCD, 6 (2.6\%) Arab athletes reported a history of syncope, and 8 $(3.8 \%)$ experienced a form of chest discomfort. Wilson et al. ${ }^{8}$ reported 17 west Asian and 14 Black athletes with a family history of SCD and 8 $(0.66 \%)$ athletes reporting cardiovascular symptoms during activity. Finally, Schmied at al. 9 shared those 36 (17\%) African athletes had a family history of SCD with 19 (9\%) indicating atypical chest discomfort.

\section{CLINICAL BOTTOM LINE}

The American Heart Association (AHA) estimates the prevalence of SCD-related abnormalities that predispose young athletes is $0.3 \%$, whereas the incidence of potentially lethal SCD consistently ranges between 0.2 to $0.7 \% .^{1}$ This correlate to 1 in 500 athletes with an undetected cardiac abnormality. ${ }^{1}$ The AHA, the ESC, and the International Olympic Committee recommend

Clinical Practice in Athletic Training Volume 4 - Issue 1 - February 2021 
cardiovascular screening for all athletes. Nevertheless, these entities do not have a uniform protocol, nor do they all advocate for the inclusion of a resting 12-lead ECG in PPE.1 Several differences exist when comparing the ESC recommendations to the 12-Element AHA recommendations for cardiac pre-participation screening. ${ }^{10}$

Based on the findings of the guiding systematic review and other supporting evidence, the sensitivity of preparticipation protocols that simply implement history questions and physical examinations do not significantly detect predisposing SCD-related abnormalities in young athletes. In a previous systematic review comparing screening strategies, the most clinically sensitive method to rule out a cardiovascular condition was an ECG $(94 \%)$ as compared to history $(20 \%)$ or physical exam (9\%).11 However, the specificity of each of these methods is similar (ECG: 93\%, history: 94\%, physical exam: 97\%).11 In a report of 115 SCD cases in young athletes, only 1 athlete $(0.9 \%)$ was accurately diagnosed with an underlying cardiovascular issue when using the history and physical examination screening model.'

Although there is a concern that ECGs produce a high number of false-positive results leading to unnecessary diagnostic testing, studies have shown that the adoption of modern, strict ECG criteria have lowered false-positive rates to $6 \%$ compared to history at $8 \%$ and physical examination at $10 \% .1,3$ The studies in this review highlight that the rate of false-positives are greater with a history and physical examination model. Another consideration is the cost component associated with the implementation of ECG to pre-participation screenings. The cost to perform the AHA-recommended cardiovascular history and physical examination is estimated at $\$ 0$ because it is done in conjunction with a PPE. ${ }^{12}$ However when an SCD-related abnormality is detected, the athlete will undergo a cardiology

Copyright $($ by Indiana State University All rights reserved. ISSN Online 2577-8188 consultation (average cost of $\$ 150$ ), potential treadmill testing (average cost of \$225), and 2D echocardiography (average cost of $\$ 350$ ) for an average cost of $\$ 500$ to evaluate an abnormality detected with the AHA-recommended screening. ${ }^{12}$ Conversely, the estimated cost to perform an ECG during mass PPE is $\$ 10$ with an average cost of $\$ 365$ to evaluate detected abnormalities. ${ }^{12}$ Cardiac abnormalities that have gone undetected in traditional PPE screenings prior to physical activity have resulted in wrongful death lawsuits. ${ }^{13}$ In 2012, the family of Ronald Rouse, an 18-yearold former Hartsville football player, were compensated $\$ 260,000$ to dismiss the wrongful death of their son from an undiagnosed cardiac arrhythmia that resulted in cardiac arrest. ${ }^{13}$ Similar instances of young athletes dying from cardiac arrest from detectable abnormalities include cases such as Kleinknecht v. Gettysburg College with the death of a lacrosse player and 15-year-old Star Ifeacho at Dunbar High School.14,15 This upstream initiative to minimize fatalities related to SCD through early detection could potentially save young lives at a low incremental cost of $\$ 10$ and negate costly wrongful death lawsuits. ${ }^{2}$ The interpretation of the guiding systematic review and supporting literature suggests that the addition of the updated criteria for 12-lead ECG in PPE could potentially positively detect SCD-related abnormalities when compared to history and physical examinations alone, especially in susceptible minority populations.

Conditions that predispose athletes to SCD are preventable if effectively managed through activity modification and medical intervention. The guiding systematic review reported that the prevalence of SCD occurred at higher rates in football and basketball as compared to soccer. We suggest that clinicians use this data to provide a comprehensive, patient-centered PPE exam that is reflective of the cultural and ethnic differences of the individuals we treat. While equal care would incorporate advanced cardiac testing for

Clinical Practice in Athletic Training Volume 4 - Issue 1 - February 2021 
all, the results of this guiding systematic review suggest, at minimum, equitable care be provided to the predisposed young individuals of African and Middle Eastern descent. However, equitable care extends beyond cardiac testing in minority populations. Athletic trainers must be cognizant how social determinants of health based on a patient's race and ethnicity may influence their health outcomes and risks. A prime example of this bias in care, involves how Blacks and other minority populations in the United States have been systemically denied mortgages or approval for leased housing in neighborhoods at higher rates than Caucasians for decades. ${ }^{16}$ This housing crisis cascaded to create an increasing educational gap where Black students are inadequately prepared for academic success because they attend poorer, disadvantaged school systems. ${ }^{16}$ Overall, this is just one example of how Blacks and African Americans have been impoverished and unhealthy because of an oppressive system established over 300 years ago that resulted in racialized institutions and further perpetuated white privilege. ${ }^{17}$

To provide patient-centered care, we must recognize how race and ethnicity, as well as other social determinants of health, may change the PPE process for some athletes as compared to others. Barkley et al. 16 discussed how diversity in athletic participation is continually increasing in the NCAA, but there is a need for sports medicine literature to address additional knowledge, skills, and abilities in cultural competency and to promote health equity. ${ }^{16}$ Historically in this nation, minority populations, especially Black people, have not been treated equitably in the healthcare system compared to their Caucasian counterparts. 16,17,18,19 Evidence also suggests that health care professionals, such as athletic trainers and team physicians, have limited knowledge about effectively delivering culturally competent care. ${ }^{16}$ This variance in treatment in minority populations, especially in Black communities, stems from the higher proportion of minorities

Copyright $($ by Indiana State University All rights reserved. ISSN Online 2577-8188 living in "medical deserts" where communities lack health care resources. ${ }^{19}$ Residential segregation and racial disparities are a fundamental cause for health disparities and have an association with infant mortality, adult mortality, poor health status, smoking during pregnancy, poor birth outcomes, tuberculosis and other infectious diseases, and exposure to cancer-causing air toxins. ${ }^{16}$ The discord in research that quantifies these statistics and the effort to combat these issues do not align. The majority-white decisionmakers include public health researchers, policymakers, medical educators, officials, hospital administrators, and insurance and pharmaceutical executives. ${ }^{17}$ Caucasians make up three quarters of those practicing medicine, $77 \%$ of American Medical Association delegates, 85\% of American Medical Association board members, 23 of 27 directors of the National Institutes of Health, $90 \%$ of National Institutes of Health branch and lab chiefs, and $83 \%$ of senior investigators. ${ }^{17}$ These healthcare providers write policies from a position of privilege, creating a White framework which normalizes discriminatory practices and reinforces institutionalized inequity in health care and health - a contributing factor in the delay to adopt cardiac screening by many leading sports organizations. ${ }^{17}$ Public health efforts to reduce SCD in Black athletes is a crucial step; however, it is only a small aspect of the health disparity issue that needs to be addressed holistically for true equality to be achieved. ${ }^{18}$ Further research is needed to understand the underlying persistent racial differences in SCDrelated abnormalities to mitigate the risk of $S C D$ in Black athletes. ${ }^{18}$

The profession of athletic training is continuously advancing its skillset to minimize patient risk of injury and illness. Athletic trainers complete PPEs annually to mitigate these risks. In the United States, the NATA suggests the implementation of the 12-Element AHA recommendations for cardiovascular screening to detect potential underlying cardiac conditions in young athletes. ${ }^{2}$ 
However, evidence indicates that questioning an athlete about their personal and family history paired with a physical examination does not accurately detect these fatal disorders. ${ }^{12} \mathrm{~A}$ parallel example of a lethal medical condition in the athletic setting is sickle cell trait, which athletic trainers now readily test for. The literature provides evidence that ECGs are highly sensitive in indicating SCD-related abnormalities and should become common practice during PPEs in both the collegiate and youth setting. These entities should strive to incorporate ECG testing into their policies and procedures as a means of best practice. The results from the manuscript suggest that additional research on the prevalence of abnormalities correlated to SCD in young Middle Eastern and African competitive athletes should be conducted since SCD disproportionately impacts athletes of color; especially young, African American males.

\section{REFERENCES}

1. Asif IM, Toresdahl BG, Drezner JA. Chapter 3: Prevention of sudden cardiac death in young athletes. In: Casa DJ, Stearns RL. Preventing Sudden Death. 2nd ed. Jones \& Bartlett Learning; 2017:3350.

2. Conley KM, Bolin DJ, Carek PJ, Konin JG, Neal TL, Violette D. National athletic trainers' association position statement: Preparticipation physical examinations and disqualifying conditions. J Athl Train. 2014;49(1): 102-120. https://doi.org//10.4085/1062-605048.6.05.

3. Winkelmann ZK, Crossway AK. Optimal screening methods to detect cardiac disorders in athletes: An evidence-based review. J Athl Train. 2017;52(1 2):1 1681170 . https://doi.org/10.4085/10626050-52.11.24.
4. Peterson DF, Kucera K, Thomas LC, Maleszewski J, Siebert D, LopezAnderson M, Zigman M, Schattenkerk J, Harmon KG, Drezner JA. Aetiology and incidence of sudden cardiac arrest and death in young competitive athletes in the USA: A 4-year prospective study. BJSM. Published Online First: 12 November 2020. https://doi.org/10.1136/bjsports2020-102666.

5. Casa DJ, Guskiewicz KM, Anderson SA, Courson RW, Heck JF, Jimenez CC, McDermott BP, Miller MG, Stearns RL, Swartz EE, Walsh KM. National athletic trainers' association position statement: Preventing sudden death in sports. J Athl Train. $2012 ; 47(1)$ :96-118. https://doi.org/10.4085/1062-605047.1.96.

6. Riding NR, Sheikh N, Adamuz C, Watt V, Faroog A, Whyte GP, George KP, Drenzer JA, Sharma S, Wilson MG. Comparison of three current sets of electrocardiographic interpretation criteria for use in screening athletes. Heart. 2015;101:384-390. https://doi.org/10.1136/heartinl-2014306437.

7. Alattar A, Ghani S, Mahdy N, Hussain H, Maffulli N. Pre-participation musculoskeletal and cardiac screening of male athletes in the United Arab Emirates. Transl Med UniSa. 2014;9:4349. PMID: 24809035.

8. Wilson MG, Chatard JC, Carre F, Hamilton B, Whyte GP, Sharma S, Chalabi H. Prevalence of electrocardiographic abnormalities in West-Asian and African male athletes. Br J Sports Med. 201 2;46:341 -347. 
https://doi.org/10.1136/bjsm.2010.08

2743.

9. Schmied C, Di Paolo FM, Zerguini AY, Dvorak J, Pelliccia A. Screening athletes for cardiovascular disease in Africa: $A$ challenging experience. $\mathrm{Br} J$ Sports Med. 2013;47(9):589-584.

https://doi.org/10.1136/bjsports2012-091803.

10. Lithwick DJ, Fordyce $C B$, Morrison $B N$, Nazzari H, Krikler G, Isserow S, Heilbron $B$, Taunton J. Pre-participation screening in the young competitive athlete: International recommendations and a Canadian perspective. B C Med J. 2016;58(3):145-151.

11. Harmon KG, Zigman M, Drezner JA. The effectiveness of screening history, physical exam, and ECG to detect potentially lethal cardiac disorders in athletes: A systematic review/metaanalysis. J Electrocardiol. 2015;48(3):329-338. https://doi.org/10.1016/i.jelectrocard. 2015.02.001.

12. Fuller CM. Cost effectiveness analysis of screening of high school athletes for risk of sudden cardiac death. Med Sci Sports Exerc. 2000;32(5):887-890.

13. Smith C. Parents settle S.C. football wrongful death suit for $\$ 260,000$. USA Today. December 14, 2016. Accessed April 2, 2021. https://usatodayhss.com/2016/parentssettle-outstanding-football-wrongfuldeath-suit-for-just-260000.

14. Kleinknecht v. Gettysburg, 786 F. Supp. 449 (M.D. Pa 1992).

15. Mother of high school athlete who died after collapsing at school files lawsuit.
WKYT. December 11, 2017. Accessed April 2, 2021.

https://www.wkyt.com/content/news/M other-of-high-school-athlete-who-diedafter-collapsing-at-school-files-lawsuit463411543.html.

16. Barkley L, Taliaferro LA, Baker K, Garcia J. The holistic athletic healthcare model: Addressing the developmental, social, and cultural needs of collegiate athletes. JHEAI. 2018;1(3):26-47. https://doi.org/10.15763/issn.23765267.2018.1.3.26-47.

17. Feagin J, Bennefield Z. Systemic racism and U.S. health care. Soc Sci Med. 2014;103:7-14. https://doi.org/10.1016/i.socscimed.20 13.09.006.

18. Deo R, Safford MM, Khodneva YA, Jannat-Khah DP, Brown TM, Judd SE, McClellan WM, Rhodes JD, Shlipak MG, Soliman EZ, Albert CM. Differences in risk of sudden cardiac death between Blacks and Whites. J Am Coll Cardiol. 2018;72(20):2431-2439. https://doi.org/10.1016/i.jacc.2018.08 .2173 .

19. Gaskin DJ, Dinwiddie GY, Chan KS, McCleary RR. Residential segregation and the availability of primary care physicians. Health Serv Res. $2012 ; 47(6): 2353-2376$. https://doi.org/10.1111/i.1475$\underline{6773.2012 .01417 .}$. 